\title{
Response to: Do not yet abandon cephalic vein access for multiple leads in ICD implantation
}

\author{
Chirag Barbhaiya ${ }^{1}$, Osama Niazi ${ }^{1}$, Lior Jankelson ${ }^{1}$, Scott Bernstein ${ }^{1}$, David Park ${ }^{1}$, Douglas \\ Holmes $^{1}$, Anthony Aizer ${ }^{1}$, and Larry Chinitz ${ }^{2}$ \\ ${ }^{1}$ NYU Langone Health \\ ${ }^{2}$ NYU Langone Medical Center New York University
}

July 29, 2020

Our article reported risk factors for ICD lead failure at our medical center, and we found an elevated risk of ICD lead failure in multiple lead ICD systems implanted via cephalic venous access.(1) Our analysis was prompted by recent literature related to durability of the Linox ICD lead (Biotronik, Inc., Berlin, Germany), and we found similar, elevated risk of ICD lead failure implanted in multiple lead systems via cephalic access in Linox and non-Linox ICD leads. Given the small number of total lead failures in the overall cohort (6 of 660), and the retrospective, single-center nature of our analysis, we reviewed prior Linox ICD lead durability manuscripts for evidence of increased risk of failure in multiple lead ICD systems implanted via cephalic venous access. While no prior manuscript evaluated this specific risk, we did find a trend towards increased risk of lead failure in cohorts with greater proportions of multiple lead systems, and greater proportions of systems implanted via cephalic access, however these variables were included in the analysis in a minority of prior studies.

Dr. Maas and colleagues express surprise at the high failure rate when implanting multiple leads in our cohort. We would clarify that we reported ICD lead failure in 4 of the 304 patients in our cohort with multiple ICD leads, and that the frequency of lead failure in multiple lead ICD systems was not statistically significantly different compared to that of single lead ICD systems. In contrast, and surprisingly to us, 3 of 30 patients with multiple lead ICD systems implanted via cephalic access experienced ICD lead failure, and the frequency of ICD lead failure was significantly greater in this group compared to the remaining cohort in Kaplan-Meier survival analyses.

Maas and colleagues question the reason for utilization of cephalic access in $18 \%$ of patients, hypothesize that suboptimal implantation technique may be responsible for the elevated lead failure rate, and request clarification of lead failure mechanism. We did not systematically collect rationale for venous access technique, and venous access techniques was at the discretion of the implanting physician. Of the 6 lead failures, 3 were related to lead noise, and 3 were related to rising pacing thresholds. Of the three lead failures amongst patients with multiple lead systems implanted via cephalic venous access, 2 were related to lead noise, and 1 was related to a rising pacing threshold. We believe that the lead noise may be related to insulation breach that may be predisposed by lead-lead interactions in the region of the cephalic vein. ICD leads were returned to the manufacturer on an ad hoc basis, and no specific feedback was received from manufacturers related to leads included in our analysis. All implanting physicians were experienced operators, and there were no significant differences in frequency of ICD lead failure by operator. We agree that implantation technique may play an important role in lead failure risk, and our analysis should prompt extra caution when implanting multiple leads via cephalic venous access.

Citing the above limitations of our analysis, Dr Maas and colleagues state that it is "too early to abandon cephalic vein access, even for multiple lead systems." They also review recent literature reporting favorable 
acute outcomes of ultrasound guided axillary venous access. We agree that our analysis paired with our literature review is best considered hypothesis generating, and we hope that our analysis encourages future studies to consider our findings when selecting variables of interest in ICD lead durability studies. We share Dr. Maas and colleagues' favorable view of data supporting axillary venous access, particularly in combination with ultrasound guidance. As a result, given the available evidence of acceptable alternative techniques, our practice is to favor axillary venous access during implantation of multiple lead ICD systems, but we would not hesitate to implant via cephalic venous access in the appropriate clinical scenario.

\section{References}

1. Barbhaiya CR, Niazi O, Bostrom J et al. Early ICD lead failure in defibrillator systems with multiple leads via cephalic access. Journal of cardiovascular electrophysiology 2020;31:1462-1469. 